Traditional Medicine and Modern Medicine

Vol. 2, No. 1 (2019) 7-11

(C) Institutes of Integrative Medicine, Fudan University and

World Century Publishing Corporation

DOI: $10.1142 /$ S2575900019500010

\title{
Experimental study on the effects of Kursi Ziyabet on the rat model of diabetes mellitus
}

\author{
Alimjan Parhat ${ }^{\S}$, Haibaier Huojiaaihemaiti ${ }^{\S}$, Nabijan Mohammadturusn ${ }^{*}$, \\ and Mammat Nurahmat ${ }^{\dagger+}$ \\ College of Xinjiang Uyghur Medicine \\ Hotan, Xinjiang 848000, P. R. China \\ Key Laboratory of Uyghur Medicine Research \\ College of Xinjiang Uyghur Medicine \\ Hotan, Xinjiang 848000, P. R. China \\ *nabijanm@126.com \\ †1830468427@qq.com
}

Received 1 November 2018; Accepted 27 December 2018; Published 1 March 2019

\begin{abstract}
Objective: To evaluate the hyperglycemic effects of Kursi Ziyabet (KZ) tablets on the rat models of diabetes mellitus.

Methods: In total, 58 male SD rats were assigned randomly to six groups. All except the normal group were transformed into experimental diabetes mellitus rat models by injecting streptozocin. The hyperglycemic effect and the mechanism of Ziyabet were evaluated by body weight, food and water intake, fasting blood sugar, and related parameters by measuring the oxidative stress-related factors and lipid metabolism indicator level by the corresponding kits using the rat experimental models.
\end{abstract}

Results: Compared with the model group, body weight markedly increased after 3-6 weeks of intragastric administration of Ziyabet tablets $(p<0.01)$, while the water intake significantly decreased in the same period of time $(p<0.05)$. Food intake and fasting blood sugar level also decreased with the high dosage of Ziyabet tablets $(p<0.05)$. There is no significant difference in pancreas' MDA content of the Ziyabet groups when compared to the model group $(p>0.05)$, while significant increase in SOD level was observed in high-dosage $\mathrm{KZ}$ group $(p<0.05)$. The blood serum insulin and free fatty acid level also decreased in the high-dosage KZ group compared with the model group $(p<0.05)$.

Conclusion: We conclude that Ziyabet tablets demonstrated protective effects on the diabetic rat models.

Keywords: Kursi Ziyabet; diabetes mellitus; rat model.

\section{Introduction}

Diabetes mellitus (DM) is one of the most prevalent clinical disorders in the world. It has been reported that there are 387 million people diagnosed with diabetes globally, and it is expected to double by $2030 .^{1,2}$ Insulin resistance is believed to be the most encountered risk factor during the development of type-2 DM. It will induce dysregulation of glucose in multiple tissues, which is the major cause of hyperglycemia and diabetes. ${ }^{3}$

With thousands of years of medical practice, a great deal of valuable experience has been accumulated in the Chinese traditional medical system for diabetes therapy. ${ }^{4}$ Traditional Uyghur medicine, as one of the main branches of Chinese medical system, has significantly contributed to the disease treatment and prevention of people in the northwest region of

¥Corresponding authors.

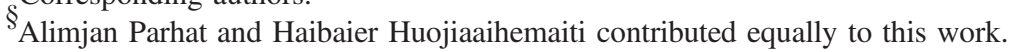


China. It has special characteristics in treating diseases like diabetes. According to the traditional Uyghur medicine, diabetes occurs when the temperament of kidney and liver changes into hot temperament and they absorb more water from the blood. Therefore, it will make diabetes patients thirsty. Kursi Ziyabet (KZ) has long been used to treat diabetes and diabetes-related syndromes among people. It has the effect of regulating Sevda and quenching the thirst. ${ }^{5,6}$ However, till now there is a lack of scientific reports on the effects of Kursi Ziyabet on diabetes. In this paper, we preliminarily studied the effects of Kursi Ziyabet on the rat models of diabetes mellitus.

\section{Material and Methods}

\section{Materials}

Kursi Ziyabet tablets are provided by the College of Xinjiang Uyghur Medicine, China (Prescription no. M2003007). The other materials/equipment used are Metformin (AAS7221), Alloxan (Sigma; 046K1206), Superoxide Dismutase (SOD) assay kit, malondialdehyde (MDA) assay kit (both from the Nanjing Jiancheng Bioengineering Institute; A001-1, A0031), Insulin Rat ELISA kit (Invitrogen), free fatty acid (FFA) assay kit (both from Beijing Jiuqiang Biotech), Roche blood glucometer (ACCU-CHEK; 475185), DY89-1 electric glass homogenizer, microplate reader (TECAN Infinite F200 PRO; Bio-Rad), and high-speed centrifuge (Beckman).

\section{Methods}

In total, 60 Sprague Dawley (SD) rats of $(180 \pm 20) \mathrm{g}$ were purchased from the Experimental Animal Center, Xinjiang Medical University, Urumqi, China [SCXK(Xin) 20160003]. The SD rats were fed ad libitum with standard commercial diet. All the rats were kept in an air-conditioned room with a 12-h light/dark cycle with free access to water and food. All animal experiments were carried out in strict accordance with the standards set by Laboratory Animal Care and Use Committee at Xinjiang Medical University (Urumqi, China) and the Guideline on the Humane Treatment of Laboratory Animals established by the People's Republic of China.

\section{Model establishment}

About 2.5\% Alloxan solution was prepared with saline water. Rats were weighed and freshly prepared $0.25-\mathrm{mL} / \mathrm{kg}$ Alloxan solution was intraperitoneally injected for two consecutive days. Normal group was injected with equal level of saline water. Their glucose levels were tested and the rats with low glucose level were treated with Alloxan solution again. After two weeks, rats with fasting blood glucose (FBG) level $\geq 11.1 \mathrm{mmol} / \mathrm{L}$ were considered to be the diabetic rat models. They were further divided into model group, lowdosage $\mathrm{KZ}$ group $(300 \mathrm{mg} / \mathrm{kg})$, medium-dosage $\mathrm{KZ}$ group $(600 \mathrm{mg} / \mathrm{kg})$, high-dosage $\mathrm{KZ}$ group $(1200 \mathrm{mg} / \mathrm{kg})$, and the Alloxan group. All drugs were intragastrically administered for three consecutive weeks, one time a day, and both the normal and model groups were intragastrically administered with saline water. The food and water intake as well as body weight were recorded every week.

After the final drug administration, rats were made to starve for $12 \mathrm{~h}$ and sacrificed under anesthesia by freshly prepared $1 \%$ pentobarbital. Blood samples were collected for serum assessment. Pancreatic tissues were removed and frozen at $-80^{\circ} \mathrm{C}$ until the analyses. SOD, MDA, insulin level in serum, and FFA level were examined according to the manufacturer's instruction.

Statistical analyses were conducted using GraphPad Prism 6.0 software. All results are presented in terms of mean ${ }^{7}$ and standard deviation (SD). Differences were determined using one-way ANOVA tests to compare the groups. A $p$-value $<0.05$ was considered statistically significant.

\section{Results}

\section{Body weight changes}

After the induction of diabetes with Alloxan, among the 60 rats, 13 rats died and two rats' FBS level was under $11 \mathrm{mmol} /$ L. So they were excluded from the experiment, as shown in Table 1. There is no significant difference in body weight between the groups $(p<0.05)$. After the third week, body weight markedly decreased in the diabetic model group as compared to the normal group $(p<0.01)$. And the body weight increased in high-dosage KZ-treated group and positive control as compared to the model group $(p<0.05$ and $p<0.01$, respectively). After the sixth week (after three weeks of treatment with $\mathrm{KZ}$ ), body weight loss was lower in both medium- and high-dosage $\mathrm{KZ}$ groups as compared to the control group $(p<0.05)$.

Table 1. Effects of Ziyabet tablets on the body weight in diabetic SD rats.

\begin{tabular}{lllll}
\hline & \multicolumn{3}{c}{ Body weight $(\mathrm{g})$} \\
\cline { 3 - 5 } Group & $N$ & Week 1 & Week 3 & Week 6 \\
\hline Normal & 8 & $231.76 \pm 10.5$ & $317.91 \pm 15.4$ & $400.97 \pm 21.2$ \\
Model & 8 & $227.37 \pm 11.8$ & $267.51 \pm 11.3^{* * *}$ & $320.61 \pm 20.5^{* * *}$ \\
Low-dosage KZ & 6 & $232.65 \pm 10.05$ & $273.36 \pm 17.4$ & $322.57 \pm 28.7$ \\
Medium-dosage KZ & 7 & $228.99 \pm 12.58$ & $280.20 \pm 11.3$ & $358.68 \pm 30.6^{\triangle}$ \\
High-dosage KZ & 8 & $233.37 \pm 8.42$ & $292.27 \pm 12.8^{\triangle}$ & $366.15 \pm 28.1^{\triangle}$ \\
Positive control & 7 & $241.84 \pm 10.42$ & $294.24 \pm 11.13^{\triangle} \triangle$ & $376.30 \pm 23.2^{\triangle} \Delta$ \\
\hline
\end{tabular}

Notes: Here, ${ }^{*} p<0.05,{ }^{* *} p<0.01$, and ${ }^{* * *} p<0.001$ indicate a statistically significant difference between the normal group and the model group; ${ }^{\triangle} p<0.05$, $\triangle \triangle p<0.01$, and $\triangle \Delta \Delta p<0.001$ indicate a statistically significant difference compared to the model group. 


\section{Food and water intake}

As shown in Tables 2 and 3, there was no significant change in both food and water intakes between the groups $(p>0.05)$. Significant change was observed in water uptake after three weeks between the normal and model groups $(p<0.01)$. And water uptake was reduced after being treated with high dosage of $\mathrm{KZ}$ in positive control as compared to the model group ( $p<0.05$ and $p<0.01$, respectively). No significant change was observed after treatment with $\mathrm{KZ}$ for one week $(p>0.05)$. However, food uptake was significantly reduced with high dosage of $\mathrm{KZ}$ after treatment for three weeks.

\section{Fasting blood glucose level}

Fasting blood glucose level was also recorded. There was a statistical meaning in the fasting blood glucose level between the model group and normal group $(p<0.01)$. And after being treated with $\mathrm{KZ}$ for one week there was no change observed in the blood glucose level $(p>0.05)$, while obvious decrease was observed in the positive control group $(p<0.01)$. High dosage of $\mathrm{KZ}$ was able to decrease blood glucose level after treatment for three weeks as compared to the model group $(p<0.01)$ (Table 4$)$.

Table 2. Effects of Ziyabet tablets on water intake in diabetic SD rats.

\begin{tabular}{lllll}
\hline & \multicolumn{3}{c}{ Water uptake $(\mathrm{mL})$} \\
\cline { 3 - 5 } Group & $N$ & Week 1 & \multicolumn{1}{c}{ Week 3 } & \multicolumn{1}{c}{ Week 6 } \\
\hline Normal & 8 & $32.14 \pm 9.8$ & $39.00 \pm 7.9$ & $43.14 \pm 9.6$ \\
Model & 8 & $30.00 \pm 10.6$ & $96.25 \pm 13.6^{* * *}$ & $98.28 \pm 16.9^{* * *}$ \\
Low-dosage KZ & 6 & $30.00 \pm 10.6$ & $97.57 \pm 12.1$ & $90.57 \pm 15.9$ \\
Medium-dosage KZ & 7 & $28.71 \pm 8.7$ & $81.42 \pm 13.5$ & $75.14 \pm 9.5$ \\
High-dosage KZ & 8 & $30.57 \pm 12.1$ & $63.29 \pm 15.4 \triangle \triangle$ & $67.14 \pm 17.5^{\triangle \triangle}$ \\
Positive control & 7 & $30.13 \pm 10.63$ & $67.14 \pm 17.54 \triangle \triangle \triangle$ & $58.57 \pm 17.27^{\triangle} \triangle$ \\
\hline
\end{tabular}

Notes: Here, ${ }^{*} p<0.05,{ }^{* *} p<0.01$, and ${ }^{* * *} p<0.001$ indicate a statistically significant difference between the normal group and the model group; ${ }^{\triangle} p<0.05$, $\triangle \triangle p<0.01$, and $\triangle \triangle \triangle p<0.001$ indicate a statistically significant difference compared to the model group.

Table 3. Effects of Ziyabet tablets on the food intake in diabetic SD rats.

\begin{tabular}{lcccl}
\hline & & \multicolumn{3}{c}{ Food intake $(\mathrm{g})$} \\
\cline { 3 - 5 } Groups & $N$ & Week 1 & Week 3 & Week 6 \\
\hline Normal & 8 & $18.37 \pm 5.4$ & $20.75 \pm 7.3$ & $25.62 \pm 5.6$ \\
Model & 8 & $17.37 \pm 3.9$ & $37.25 \pm 7.6^{* * *}$ & $39.25 \pm 6.73^{* * *}$ \\
Low-dosage KZ & 6 & $19.12 \pm 7.3$ & $40.6 \pm 8.4$ & $39.00 \pm 6.34$ \\
Medium-dosage KZ & 7 & $16.75 \pm 5.3$ & $32.62 \pm 5.8$ & $35.00 \pm 4.3$ \\
High-dosage KZ & 8 & $15.88 \pm 3.1$ & $30.37 \pm 5.4$ & $30.12 \pm 17.5^{\triangle}$ \\
Positive control & 7 & $17.13 \pm 5.1$ & $26.06 \pm 5.2^{\triangle}$ & $27.00 \pm 6.5^{\triangle}$ \\
\hline
\end{tabular}

Notes: Here, $* p<0.05, * * p<0.01$, and $* * * p<0.001$ indicate a statistically significant difference between the normal group and the model group; ${ }^{\triangle} p<0.05,{ }^{\Delta} p<0.01$, and ${ }^{\Delta} \triangle \triangle_{p}<0.001$ indicate a statistically significant difference compared to the model group.
Table 4. Effects of Ziyabet tablets on the fasting blood glucose level in diabetic SD rats.

\begin{tabular}{lcccc}
\hline & & \multicolumn{3}{c}{ Fasting blood glucose (mmol/L) } \\
\cline { 3 - 5 } Group & $N$ & Week 1 & Week 3 & Week 4 \\
\hline Normal & 8 & $5.32 \pm 1.68$ & $5.11 .86 \pm 0.79$ & $4.86 \pm 0.79$ \\
Model & 8 & $18.15 \pm 3.31^{* * *}$ & $19.81 \pm 2.71^{* * *}$ & $18.3 \pm 3.41^{* * *}$ \\
Low-dosage KZ & 6 & $17.35 \pm 3.82$ & $17.85 \pm 3.96$ & $17.16 \pm 3.53$ \\
Medium-dosage KZ & 7 & $19.55 \pm 4.68$ & $16.35 \pm 3.76$ & $14.41 \pm 2.71$ \\
High-dosage KZ & 8 & $20.14 \pm 5.72$ & $15.82 \pm 2.68$ & $13.10 \pm 2.14^{\triangle \Delta}$ \\
Positive control & 7 & $17.62 \pm 4.13$ & $14.72 \pm 3.62^{\triangle}$ & $11.82 \pm 1.80^{\triangle \Delta \Delta}$ \\
\hline
\end{tabular}

Notes: Here, $* p<0.05, * * p<0.01$, and $* * * p<0.001$ indicate a statistically significant difference between the normal group and the model group; ${ }^{\triangle} p<0.05$, $\triangle \triangle p<0.01$, and $\triangle \Delta \Delta p<0.001$ indicate a statistically significant difference compared to the model group.

Table 5. Effects of Ziyabet tablets on the MDA and SOD levels in diabetic SD rats.

\begin{tabular}{llll}
\hline Group & $N$ & MDA level & \multicolumn{1}{c}{ SOD level } \\
\hline Normal & 8 & $2.86 \pm 1.12$ & $27.83 \pm 7.49$ \\
Model & 8 & $6.16 \pm 2.59^{* *}$ & $12.82 \pm 3.33^{* * *}$ \\
Low-dosage KZ & 6 & $6.66 \pm 1.90$ & $12.51 \pm 5.18$ \\
Medium-dosage KZ & 7 & $4.83 \pm 1.57$ & $16.95 \pm 4.03$ \\
High-dosage KZ & 8 & $5.01 \pm 1.53$ & $21.15 \pm 3.96^{\triangle}$ \\
Positive control & 7 & $4.21 \pm 1.25$ & $20.62 \pm 3.42^{\triangle}$ \\
\hline
\end{tabular}

Notes: Here, $* p<0.05,{ }^{*} p<0.01$, and $* * * p<0.001$ indicate a statistically significant difference between the normal group and the model group; ${ }^{\triangle} p<0.05,{ }^{\Delta} p<0.01$, and $\triangle^{\Delta} \triangle_{p}<0.001$ indicate a statistically significant difference compared to the model group.

\section{MDA and SOD levels}

Pancreas' MDA and SOD levels were also studied. MDA level was increased in the model group while its SOD level decreased as compared to the normal group $(p<0.01)$. No change was observed in the MDA level after treatment with $\mathrm{KZ}(p>0.05)$. However, the SOD level increased after being treated with high dose of $\mathrm{KZ}$ in comparison with the model group $(p<0.05)$ (see Table 5).

\section{Blood insulin and free fatty acid levels}

As shown in Table 6, blood insulin, insulin resistance index, and free fatty acid content in diabetic rats were also recorded. Both insulin and free fatty acid levels were increased in the model group $(p<0.01)$. After being treated with $\mathrm{KZ}$, both the insulin and free fatty acid levels decreased when compared to the model group $(p<0.05)$.

\section{Discussion}

DM is a metabolic disease characterized by elevated blood sugar caused by insulin deficiency. In recent years, the incidence and prevalence of DM have been increasing year by 
Table 6. Effects of Kursi Ziyabet tablets on the blood insulin and free fatty acid levels

\begin{tabular}{lcll}
\hline Group & $N$ & Insulin $(\mathrm{mIU} / \mathrm{L})$ & FFA $(\mathrm{mmol} / \mathrm{L})$ \\
\hline Normal & 8 & $14.17 \pm 3.22$ & $0.28 \pm 0.06$ \\
Model & 8 & $41.80 \pm 4.65^{* * *}$ & $0.97 \pm 0.14^{* *}$ \\
Low-dosage KZ & 6 & $38.16 \pm 5.38$ & $0.72 \pm 0.28$ \\
Medium-dosage KZ & 7 & $33.60 \pm 4.27$ & $0.68 \pm 0.31$ \\
High-dosage KZ & 8 & $31.51 \pm 5.57^{\triangle}$ & $0.66 \pm 0.17$ \\
Positive control & 7 & $28.14 \pm 6.06^{\triangle} \triangle \triangle$ & $0.67 \pm 0.15$ \\
\hline
\end{tabular}

Notes: Here, $* p<0.05, * * p<0.01$, and $* * * p<0.001$ indicate a statistically significant difference between the normal group and the model group; $\triangle_{p}<0.05, \triangle \triangle_{p}<0.01$, and $\triangle \triangle \Delta_{p}<0.001$ indicate a statistically significant difference compared to the model group.

year, which has become a major social problem which exerts a huge toll on the physical, mental, and social well-being of affected patients. ${ }^{7-9}$ Clinically, it results in excessive drinking and eating, weight loss, polyuria, and chronic hyperglycemia as common features. ${ }^{10}$ In the present study, we also found a decrease in body weight and an increase in the food and water intake and fasting blood glucose level in the model group of diabetes. And the symptoms get reversed after being treated with $\mathrm{KZ}$, indicating that $\mathrm{KZ}$ improves diabetes-related syndromes. Moreover, KZ is designed to treat diabetes with the herbal components which are dry and cold including Bambusa textilis McClure, Luctuca sativa L., Portulaca oleracea L., Coriandrum sativum L., and Cinnamomumcamphora (L.) PreslLauruscamphora L. Based on Uyghur medicine theory, these herbs endow $\mathrm{KZ}$ to change the abnormally hot temperature of kidney and liver and thereby less water to be absorbed from the blood, so that it can improve the syndrome related with thirst. In addition, these herbs improve the liver function and increase its capability of glycogen synthesis and decomposition. Therefore, fasting blood glucose level was also decreased significantly after being treated with KZ.

In this work, we also found that $\mathrm{KZ}$ significantly reduced the levels of insulin. Moreover, there is a reduction in hyperglycemia which causes lipotoxicity damage by increasing the steatolysis and FFA level, making them to accumulate in the liver and blood. ${ }^{11}$ In our study, blood insulin level was decreased after being treated with KZ. However, there is not any change in the FFA level.

MDA is thought to be a marker useful in evaluating the degree of lipid peroxidation. ${ }^{12}$ SOD is responsible for removing oxyradicals in cells, whose activity may represent the degree of tissue damage. In the present study, pancreas' MDA and SOD levels were examined. KZ was unable to decrease the increased MDA content while it significantly increased the SOD level. A further study is needed to evaluate the antioxidant capacity of KZ.

According to traditional Uyghur medicine, a body remains in a healthy state under the condition of humors which help to keep the body in balance. If the balance was broken between the four styles of humor disease will occur. ${ }^{13}$ Therefore, the principle of treatment of diabetes should be to expel abnormal humors. ${ }^{14}$ Herbal components of $\mathrm{KZ}$ are usually cold and dry in nature. Cold and dry herbs are able to regulate liver function. Thus we hypothesize that $\mathrm{KZ}$ exerts hypoglycemic effects and intervenes in the levels of insulin in diabetic rats by enhancing the expression of related factors of insulin signaling pathway in liver and kidney. Further study should be focused on the above.

\section{Acknowledgments}

This project was funded by the Grants from Scientific Research Program of the Higher Education Institution of Xinjiang, China (XJEDU2014S088 and XJEDU20141067), Research Projects on the Study of Integrated Traditional Chinese Medicine and Western Medicine, Xinjiang Uyghur Autonomous Region, China (Grant No. 2017-03-11) and the Research Projects on Youth Science and Technology Talents Training Program of Xinjiang Uyghur Autonomous Region (QN2016YX0068).

\section{References}

1. Cassidy F, Ahearn E, Carroll BJ. Elevated frequency of diabetes mellitus in hospitalized manic-depressive patients. Am J Psychiatry 1999;156(9):1417-20.

2. Etxeberria U, de la Garza AL, Campión J, Martínez JA, Milagro FI. Antidiabetic effects of natural plant extracts via inhibition of carbohydrate hydrolysis enzymes with emphasis on pancreatic alpha amylase. Expert Opin Ther Targets 2012;16:269-97.

3. Liu Q, Liu S, Gao L, et al. Anti-diabetic effects and mechanisms of action of a Chinese herbal medicine preparation JQ-R in vitro and in diabetic $\mathrm{KK}^{\mathrm{Ay}}$ mice. Acta Pharm Sin B 2017;7 (4):461-9.

4. Li WL, Zheng HC, Bukuru J, De Kimpe N. Natural medicines used in the traditional Chinese medical system for therapy of diabetes mellitus. J Ethnopharmacol 2004;92(1):1-21.

5. Pharmacopoeia Committee of the Ministry of Health of the People's Republic of China. In: Pharmaceutical Standards of the Ministry of Health of the People's Republic of China. Ürümqi: Xinjiang Science and Technology Health Publishing House, 1999:162.

6. Li G, Luo X. Experimental study on acute toxicity of Jiangtang Ziyabiti tablets. Xinjiang Med Sci 2012;42(2):12-3.

7. Wu X, Luo Y, Xu J, et al. TCM constitutional characteristics of type 2 diabetes mellitus and its relationship with insulin resistance and secretion. J Guangzhou Univ Tradit Chin Med 2013;30(3):312-7.

8. Bradley C, Speight J. Patient perceptions of diabetes and diabetes therapy: Assessing quality of life. Diabetes Metab Res Rev 2002;18:S64-9.

9. Koopmanschap M, CODE-2 Advisory Board. Coping with Type II diabetes: The patient's perspective. Diabetologia 2002;45:S18-22. 
10. Sano R, Miki T, Suzuki Y, et al. Analysis of the insulin-sensitive phosphodiesterase $3 \mathrm{~B}$ gene in type 2 diabetes. Diabetes Res Clin Pract 2001;54:79-88.

11. Liu L, Tang D, Zhao H, Xin X, Aisa HA. Hypoglycemic effect of the polyphenols rich extract from Rose rugosa Thunb on high fat diet and STZ induced diabetic rats. J Ethnopharmacol 2017;200:174-81.

12. Jing W, Chunhua M, Shumin W. Effects of acteoside on lipopolysaccharide-induced inflammation in acute lung injury via regulation of $\mathrm{NF}-\kappa \mathrm{B}$ pathway in vivo and in vitro. Toxicol
Appl Pharmacol 2015;285:128-35.

13. Aizezi R, Abudureheman S, Wei J, et al. Uygur medical classification of complex diseases and prethrombotic state. Chin J Tradit Chin Med Inf 2009;16(2):23-5.

14. 买买提哈斯木, 艾和买提江. 维吾尔医对糖尿病足 病变的治疗和认识. 中国民族医药杂志 2001;2001(1): 14-6 [Maimaitikasimu, Aihemaitijiang. Uygur medical treatment and recognition of diabetic foot disease. Chin J Ethn Med 2001;2001(1):14-6]. 